\title{
Higher efficacy and complete response with administration of eribulin for recurrent squamous cell breast carcinoma: A case report
}

\author{
YOSHINARI GOTO, TAKASHI YOSHIDA and MORIHIKO KIMURA \\ Department of Breast Surgery, Ota Memorial Hospital, Otashi, Gunma 373-8585, Japan
}

Received June 20, 2018; Accepted July 19, 2018

DOI: $10.3892 /$ mco.2018.1676

\begin{abstract}
Squamous cell carcinoma is a rare histological type of breast cancer classified as metaplastic carcinoma. Metaplastic carcinoma involves differentiation of the breast glandular duct cells into mesenchymal tissues. While the chemotherapy regimen for metaplastic carcinoma is often similar to that for invasive ductal carcinoma, recurrence is associated with a poor prognosis due to the inadequate therapeutic efficacy of the regimen. We herein present the case of a 42-year-old female patient who underwent mastectomy of the right breast and right axillary node lymphadenectomy for T4N3aM0 breast cancer stage. The cancer was histopathologically diagnosed as squamous cell carcinoma of the breast. Adjuvant cyclophosphamide-epirubicin-fluorouracil (CEF) postoperative chemotherapy was administered, and lymphadenectomy of right-sided parasternal lymphatic metastases with pleural drainage was subsequently performed. Radiotherapy was administered to the thoracic wall and supraclavicular lymph nodes at $60 \mathrm{~Gy}$.Positron emission tomography (PET)-computed tomography (CT) examination 3 months after the radiotherapy identified accumulation of fluorodeoxyglucose (FDG) in a supraclavicular lymph node and the thoracic wall; hence, a chemotherapeutic regimen with eribulin was initiated. At 11 months after initiation of eribulin, complete response was achieved, indicated by the absence of FDG accumulation in both the supraclavicular lymph node and the thoracic wall on PET-CT. The treatment efficacy of eribulin is considered to be a result of the mixed morphology of squamous cell carcinoma, including the presence of an epithelial component, such as
\end{abstract}

Correspondence to: Dr Yoshinari Goto, Department of Breast Surgery, Ota Memorial Hospital, 455-1 Oshimacho, Otashi, Gunma 373-8585, Japan

E-mail: yoshinari510z@gmail.com

Abbreviations: CEF, cyclophosphamide-epirubicin-fluorouracil; FDG-PET/CT, fluorodeoxyglucose positron emission tomographycomputed tomography; EMT, epithelial-to-mesenchymal transition; TGF- $\beta$, transforming growth factor- $\beta$

Key words: breast cancer, chemotherapy, squamous carcinoma, metaplastic carcinoma, eribulin adenocarcinoma cells, and a mesenchymal component, in the form of sarcomatoid cells. Eribulin displayed an effect similar to that of adriamycin against malignant soft tissue tumors and was shown to effectively target mesenchymal components. In cases of reduced expression of the DNA repair pathway components, such as in metaplastic carcinomas, eribulin may be more effective compared with adriamycin, the mechanism of action of which involves inhibition of DNA synthesis. A superior therapeutic effect was obtained with eribulin in squamous cell carcinoma. Therefore, eribulin appears to be a promising, effective therapeutic choice for the management of metaplastic carcinomas, including squamous cell carcinomas.

\section{Introduction}

Squamous cell carcinoma is a type of metaplastic carcinoma; metaplastic carcinoma is characterized as carcinoma of the breast ductal tissue and stratified squamous epithelial tissue followed by their consequent differentiation into bone, cartilage and osteocartilaginous tissue. It has been reported that metaplastic breast carcinoma accounts for $<1 \%$ of all breast carcinomas, and it is characterized by large size, low incidence of lymphatic metastasis and high incidence of triplenegative phenotype (1-3). While chemotherapy for metaplastic carcinoma is commonly similar to that of invasive ductal carcinoma, a number of studies in the literature report a poor response to conventional chemotherapy $(1,4,5)$. We herein report our experience with eribulin administration in a case of recurrent squamous cell carcinoma of the breast and discuss the relevant literature.

\section{Case report}

A 42-year-old woman with carcinoma of the right breast (T4N3aM0, stage IIIC) underwent mastectomy and level III axillary lymph node dissection (Fig. 1).

Pathological examination revealed squamous cell carcinoma negative for estrogen receptor, progesterone receptor and human epidermal growth factor receptor 2 (Fig. 2). Six cycles of postoperative adjuvant chemotherapy with cyclophosphamide $500 \mathrm{mg} / \mathrm{m}^{2}$, epirubicin $75 \mathrm{mg} / \mathrm{m}^{2}$ and fluorouracil $500 \mathrm{mg} / \mathrm{m}^{2}$ (CEF regimen) were administered, following which an increase in the size of the ipsilateral sternal lymph nodes was observed. No other metastatic lesions were 

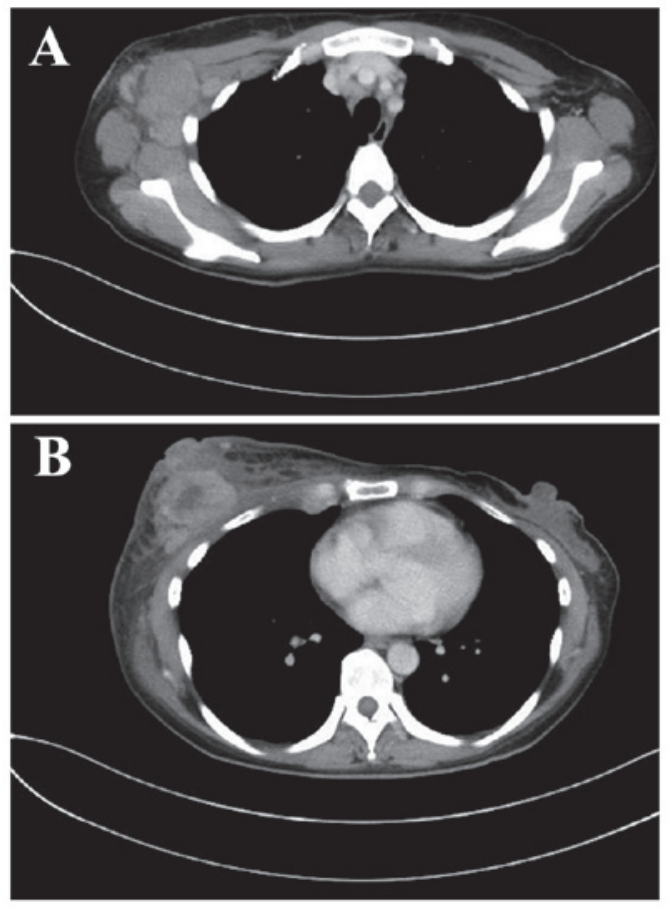

Figure 1. Contrast-enhanced computed tomography examination prior to therapy revealed (A) metastasis to the axillary lymph nodes and (B) skin invasion by the tumor cells.

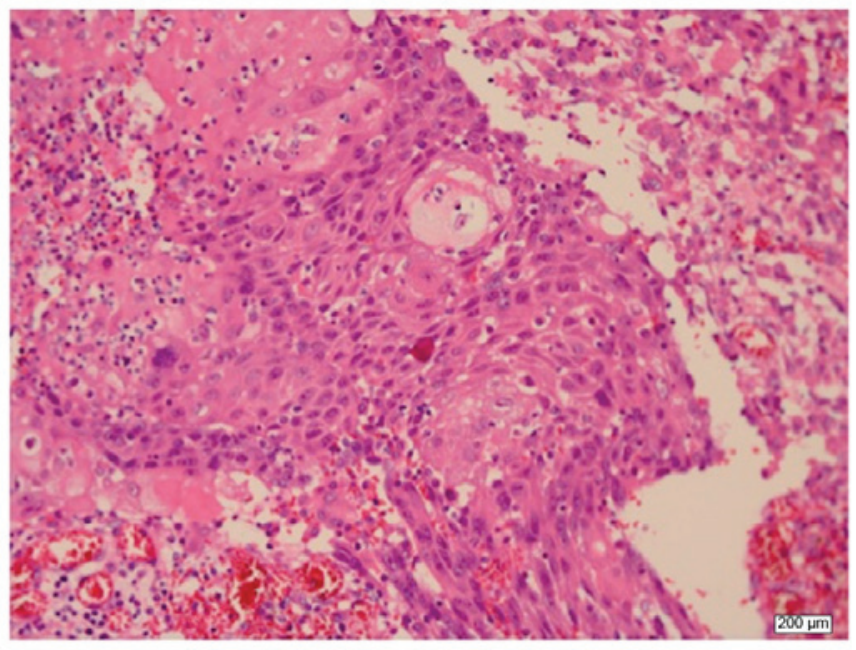

Figure 2. Pathological examination revealed that the tumor was composed of acidophilic cells with intercellular bridges. Hematoxylin and eosin staining; magnification, $\mathrm{x} 200$.

found on computed tomography (CT) scans, and the patient was subjected to lymphadenectomy with pleural drainage. Pathological examination of the resected nodes demonstrated metastatic squamous cell carcinoma. Docetaxel $\left(75 \mathrm{mg} / \mathrm{m}^{2}\right)$ was administered as adjuvant therapy; however, allergic symptoms developed, including respiratory distress and palpitations, and docetaxel was discontinued. Radiotherapy to the right thoracic wall and the right supraclavicular lymph node (60 Gy) was next administered. However, enlargement of the ipsilateral supraclavicular lymph node was observed 3 months after radiotherapy. Lymphadenopathy (lymph node enlarged to $2 \mathrm{~cm}$ in diameter) was identified on ultrasound examination,
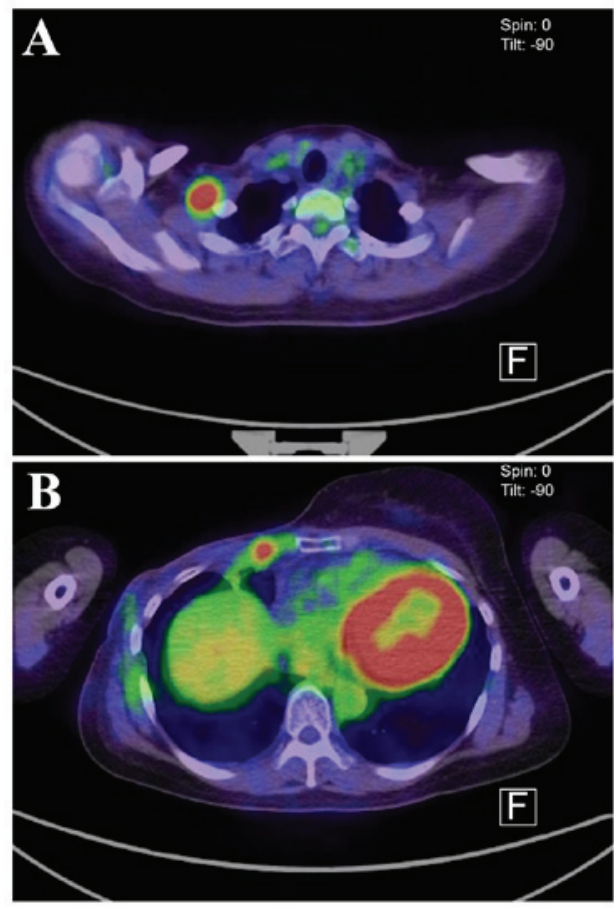

Figure 3. Accumulation of fluorodeoxyglucose in (A) a supraclavicular lymph node and (B) the anterior thorax near the previous surgical site were confirmed by positron emission tomography-computed tomography prior to the initiation of chemotherapy.
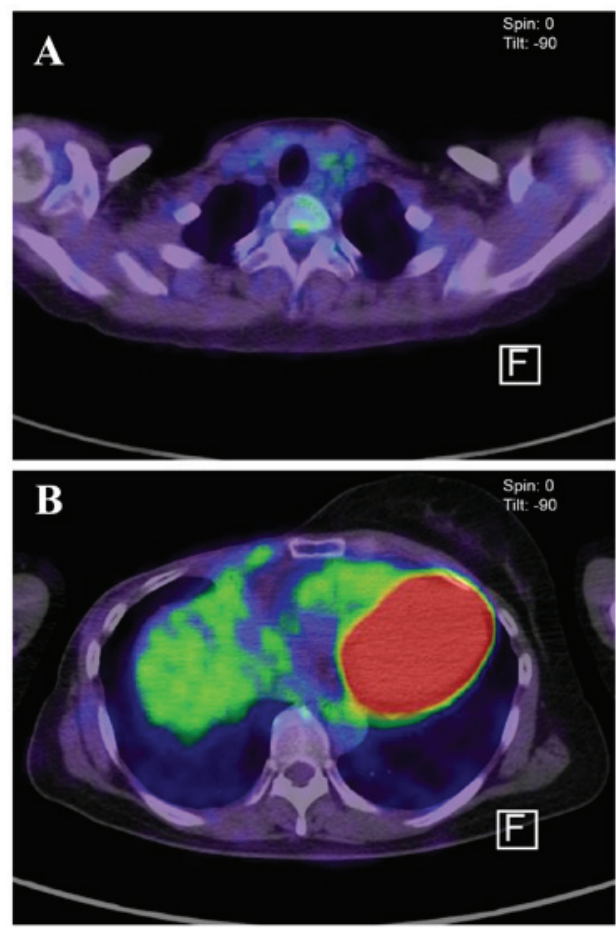

Figure 4. Positron emission tomography-computed tomography scan at 11 months after the initiation of chemotherapy confirmed the disappearance of FDG accumulation at (A) supraclavicular lymph node and (B) anterior thorax of previous surgical site.

and fine-needle aspiration cytology confirmed the presence of metastatic squamous cell carcinoma. Fluorodeoxyglucose positron emission tomography (FDG-PET)-CT revealed 
accumulation in the anterior thoracic wall close to the previous surgical site (Fig. 3). Consequently, eribulin $\left(1.4 \mathrm{mg} / \mathrm{m}^{2}\right)$ was administered as initial therapy. The lymph node was not palpable at 1 month after therapy initiation, and at 3 months after chemotherapy the enlargement had completely disappeared, as demonstrated on a CT examination. No metastatic lymph node or thoracic wall FDG accumulation was observed on PET-CT 11 months after treatment initiation (Fig. 4). After 17 months of treatment, the patient remained metastasis-free. During those 17 months, no major side effects were observed although peripheral neuropathy not affecting daily life grade 1 peripheral neurotoxicity occurred.

\section{Discussion}

It is generally recommended that the protocols for the treatment of invasive ductal carcinoma of breast cancer are applied for the treatment of squamous cell carcinoma. However, poor efficacy of chemotherapy in recurrent carcinomas, including squamous cell carcinoma, has been reported to date. While previous studies have reported the use of anthracyclines and taxanes, to the best of our knowledge, there are yet no published reports on the efficacy of eribulin. We herein discuss the possible reasons for the observed effectiveness of eribulin in the treatment of metaplastic breast carcinoma.

In addition to its effectiveness against the epithelial component, it is possible that eribulin is also effective against the mesenchymal component of the carcinoma. Drug regimens including adriamycin, which is also indicated for soft tissue tumors with a mesenchymal component, have been reported to significantly prolong progression-free survival in metaplastic carcinomas compared with other regimens. Reduced incidence of hepatic metastasis has also been reported with the use of ifosfamide and etoposide, which are used for treating soft tissue tumors (6). In Study 309, eribulin was found to be effective against liposarcoma or leiomyosarcoma (7). Eribulin promotes DNA repair, the inhibition of which is characteristic of metaplastic carcinomas, and is thus considered a more effective therapeutic agent compared with adriamycin, of which the primary mechanism of action involves the inhibition of DNA synthesis.

Furthermore, in metaplastic carcinomas, tumors including squamous epithelial and sarcomatous components exhibit increased expression of TWIST and SNAIL 2 transcription factors, suggesting an association with epithelial-tomesenchymal transition (EMT) $(8,9)$. Reports indicate that the occurrence of EMT in cancer cells coincides with the development of resistance to various anticancer agents. Several anticancer agents have been reported to induce EMT and are considered to be involved in drug resistance. Albeit in an in vitro study, eribulin has been shown to inhibit Smad 2 and Smad 3 phosphorylation via transforming growth factor- $\beta$ induction or MET inhibition. These characteristic mechanisms of action of eribulin are considered to contribute to the effectiveness of the therapy in the present case.

\section{Acknowledgements}

The authors would like to thank Shiro Sugihara for the pathological examinations and interpretations.

\section{Funding}

No funding was received.

\section{Availability of data and materials}

All data generated or analyzed during this study are included in this published article.

\section{Authors' contributions}

YG designed the study and wrote the initial draft of the manuscript. TY contributed to the analysis and interpretation of data and assisted in the preparation of the manuscript. MK critically reviewed the manuscript. All authors have read and approved the final version of the manuscript.

\section{Ethics approval and consent to participate}

The study was conducted in accordance with the principles of the Declaration of Helsinki.

\section{Patient consent for publication}

This patient and her family were informed that the data from her case would be submitted for publication and provided written informed consent.

\section{Competing interests}

The authors declare that they have no competing interests.

\section{References}

1. Rayson D, Adjei AA, Suman VJ, Wold LE, Ingle JN: Metaplastic breast cancer: prognosis and response to systemic therapy. Ann Oncol 10: 413-419, 1999.

2. Pezzi CM, Patel-Parekh L, Cole K, Franko J, Klimberg VS, Bland K: Characteristics and treatment of metaplastic breast cancer: analysis of 892 cases from the National Cancer Data Base. Ann Surg Oncol 14: 166-173, 2007

3. Lee H, Jung SY, Ro JY, Kwon Y, Sohn JH, Park IH, Lee KS, Lee S, Kim SW, et al: Metaplastic breast cancer: clinicopathological features and its prognosis. J Clin Pathol 65: 441-446, 2012.

4. Chen IC, Lin CH, Huang CS, Lien HC, Hsu C, Kuo WH, Lu YS, Cheng AL, et al: Lack of efficacy to systemic chemotherapy for treatment of metaplastic carcinoma of the breast in the modern era. Breast Cancer Res Treat 130: 345-351, 2011.

5. Abouharb S, Moulder S: Metaplastic breast cancer: clinical overview and molecular aberrations for potential targeted therapy. Curr Oncol Rep 17: 431, 2015.

6. Brown-Glaberman U, Graham A, Stopeck A: A case of metaplastic carcinoma of the breast responsive to chemotherapy with Ifosfamide and Etoposide: improved antitumor response by targeting sarcomatous features. Breast J 16: 663-665, 2010

7. Schoffski P, Chawla S, Maki RG, Italiano A, Gelderblom H, Choy E, Grignani G, Camargo V, Bauer S, et al: Eribulin versus dacarbazine in previously treated patients with advanced liposarcoma or leiomyosarcoma: a randomised, open-label, multicentre, phase 3 trial. Lancet 387: 1629-1637, 2016.

8. Karreth F, Tuveson DA: Twist induces an epithelial-mesenchymal transition to facilitate tumor metastasis. Cancer Biol Ther 3: 1058-1059, 2004.

9. Valsesia-Wittmann S, Magdeleine M, Dupasquier S, Garin E, Jallas AC, Combaret V, Krause A, Leissner P, Puisieux A: Oncogenic cooperation between H-Twist and N-Myc overrides failsafe programs in cancer cells. Cancer Cell 6: 625-630, 2004. 